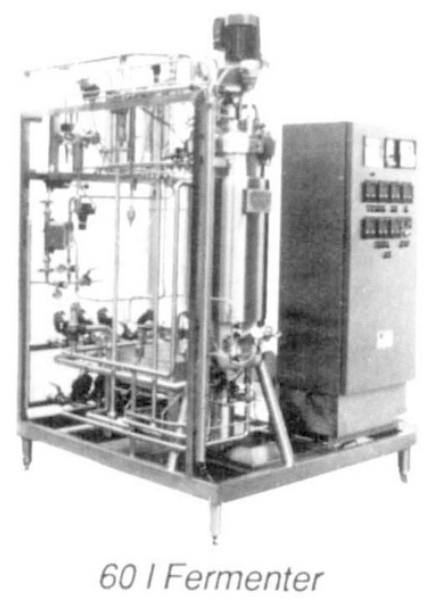

PIERRE GUERIN

Complete range of fermentation and cell culture systems

Turn key plants

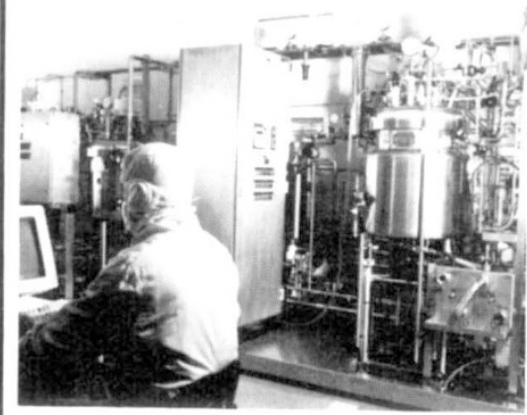

Continuous production of monoclonal antibodies

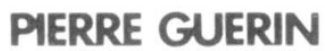

Project engineering High tech automation Manufacturing

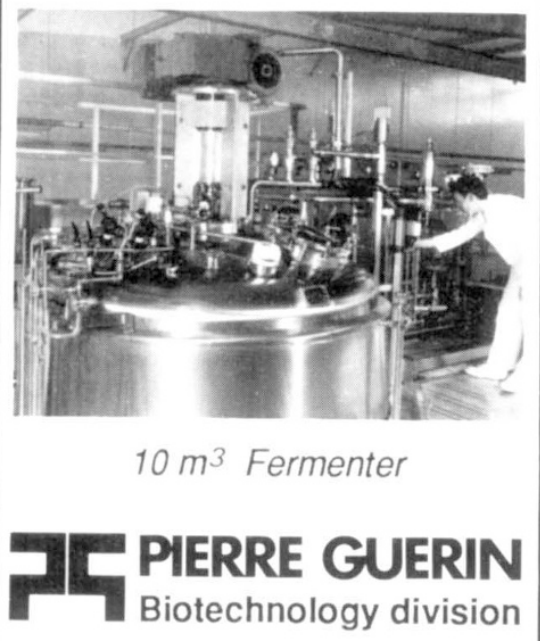

B.P. 12 - 79210 MAUZE France Tel. (33) 49.26.30.58

Fax. (33) 49.26.34.84

\title{
Will genetic tests and computers invade privacy?
}

WASHINGTON, D.C.--In a recent report that analyzes the social and policy implications of new genetic tests, voluntary participation and the continued right to privacy are among the key principles set forth. Entitled "Assessing Genetic Risks: Implications for Health and Social Policy," the report is authored by the Institute of Medicine (IOM, Washington, DC). Yet computerized medical records and electronic transmission of medical data make it increasingly difficult to assure that genetic or other medical information can be kept private, according to another recent report, this one authored by the Congressional Office of Technology Assessment (OTA, Washington, DC) and entitled "Protecting Privacy in Computerized Medical Information."

The IOM report points out that both public and private research programs are leading to an increased diversity and availability of genetic tests. Genetic testing is done to serve diverse medical goals. These test are used, for example, as prenatal tests, to screen newborns, for direct medical diagnosis, to test carriers of genetic traits, and to assess an individual's risk for developing a particular disease.

The genetic information gathered can be abused, according to the IOM. "Pressure, especially from those with commercial interests, is being exerted to broaden screening programs and increase the number of genetic tests available to the public. Before safeguards are in place, the information could be misused or misinterpreted. Among the results could be loss of insurance or employment," states the IOM.

One precautionary measure recommended by the IOM is to conduct "pilot studies" to validate genetic tests before they are introduced into routine use. Once these tests are approved, it is important to apply "the highest level of federal oversight" to assure quality control, the IOM asserts, adding that "genetic tests warrant a standard with a close-to-zero margin of error."

The proper use and interpretation of genetic tests will also depend on improving the knowledge of physicians and counselors who deal with genetic issues, as well as on improving the knowledge of the general public. The ability of individuals and families to make "informed consent" decisions based on results from increasingly complex batteries of genetic tests will be enhanced if public education about genetics is improved, the IOM asserts.

In addition to insisting on better education and on high performance for manufacturers developing genetic tests and laboratories performing such tests, the IOM declares that four ethical and legal principlesautonomy, confidentiality, privacy, and equity - should "anchorefforts to set policies" for genetic testing.

"We also strongly uphold nondirective advice," says Arno Motulsky, who chaired the IOM committee that wrote the genetictesting report and who is a professor of medicine and genetics at the University of Washington (Seattle, WA). "We recommend not telling patients what to do with genetic-test results, but leaving it up to them to decide." He and other IOM committee members also strongly recommend that "genetic information not be used to discriminate in health insurance or employment."

For its part, the OTA report points out how computers are making it more difficult to safeguard the confidentiality of medical information, such as that obtained through new genetic tests. Although computers offer opportunities to streamline the health-care system, the "challenge is to secure and maintain individual privacy, while making appropriate information available for approved uses," states the OTA.

"The present legal system, a patchwork of state laws, does not take into account that, in a computerized system, information will frequently and easily cross state borders and will therefore be subject to inconsistent legal standards," asserts the OTA. It concludes that "federal legislation is necessary" to prevent misuse of confidential medical information and to preserve patient privacy.

—Jeffrey L. Fox 